\title{
Nota Científica:
}

\section{REDESCOBERTA E TIPIFICAÇÃO DE OCTOMERIA LEPTOPHYLLA Barb. Rodr. (ORCHIDACEAE), MICRO-ORQUídEA ENDÊMICA DE Minas Gerais, Brasil}

\author{
Luiz Menini Neto ${ }^{1,2}$ \& Americo Docha Neto ${ }^{3}$
}

\begin{abstract}
RESUMO
(Redescoberta e tipificação de Octomeria leptophylla Barb. Rodr. (Orchidaceae), micro-orquídea endêmica de Minas Gerais, Brasil) A redescoberta de Octomeria leptophylla, micro-orquídea endêmica do estado de Minas Gerais, previamente considerada extinta, é aqui relatada. João Barbosa Rodrigues, em 1877, descreveu O. leptophylla baseado em material proveniente da Serra de Caldas (município de Caldas), sul de Minas Gerais. Desde então, não houve novos registros da espécie em herbários ou coleções particulares. Após 127 anos de sua descrição, expedições recentes localizaram populações de $O$. leptophylla nos municípios de Andradas e Poços de Caldas, adjacentes à localidade-tipo. Tal fato, contudo, não diminui a urgência de sua conservação. A perda de habitats e o endemismo extremo contribuem para sua categorização como espécie criticamente em perigo com base nos critérios da IUCN.
\end{abstract}

Palavras-chave: conservação, floresta atlântica, extinção local, microendemismo, taxonomia.

\section{Abstract}

(Rediscovery and typification of Octomeria leptophylla Barb. Rodr. (Orchidaceae), an endemic micro-orchid from Minas Gerais, Brazil) The rediscovery of Octomeria leptophylla, an endemic micro-orchid from Minas Gerais, Brazil thought to be extinct is presented. In 1877, João Barbosa Rodrigues described O. leptophylla based on a collection from "Serra de Caldas" (Municipality of Caldas), south of Minas Gerais. Since then, no other records were made either by herbarium botanists or private collector, and the species has been treated as extinct. During recent field expeditions to the region where the type was collected, 127 years after its description, populations of $O$. leptophylla were located at the municipalities of Andradas and Poços de Caldas. Unfortunately, the rediscovery does not mean that the species is out of danger risks from habitat loss and its restricted endemism place it as Critically Endangered according to the criteria of the IUCN.

Key-words: conservation, atlantic forest, local extinction, microendemism, taxonomy.

O gênero Octomeria R. Br. compreende aproximadamente 150 espécies, distribuídas na região Neotropical, desde Cuba e Antilhas até o norte da Argentina, sendo as Regiões Sul e Sudeste do Brasil importantes centros de diversidade. Pertence à subtribo Pleurothallidinae, a maior dentre as Orchidaceae, com mais de 4000 espécies conhecidas (Luer 1986; Pridgeon \& Chase 2001; Pridgeon et al. 2005).

De modo geral as espécies dessa subtribo, conhecidas popularmente como micro-orquídeas, não apresentam grande apelo ornamental em virtude tanto das dimensões reduzidas das plantas e suas flores como das dificuldades de cultivo, minimizando a pressão exercida pelos colecionadores. Por outro lado, muitas de suas espécies estão restritas, no Brasil, a um dos biomas mais ameaçados do planeta, a floresta atlântica, considerada um dos "hotspots" mundiais de biodiversidade, definidos como regiões que concentram grande riqueza de espécies e endemismos, mas que tiveram sua área original reduzida em mais de 70\% (Myers et al. 2000).

Octomeria leptophylla foi descrita por Rodrigues (1877) baseado em material

Artigo recebido em 11/2008. Aceito para publicação em 03/2009.

${ }^{1}$ Centro de Ensino Superior de Juiz de Fora, Campus Arnaldo Jannsen, Luz Interior, 345, Santa Luzia, 36030-776, Juiz de Fora, Minas Gerais. Autor para correspondência: menini_neto@ hotmail.com

${ }^{2}$ Doutorando da Escola Nacional de Botânica Tropical do Instituto de Pesquisas Jardim Botânico do Rio de Janeiro.

${ }^{3}$ Projeto Orchidstudium, R. Maranhão 111, 37701-025, Poços de Caldas, Minas Gerais. docha@orchidstudium.com 
proveniente de localidade próxima a Pedra Branca, município de Caldas, região sul do estado de Minas Gerais (Fig. 1), um afloramento granítico com vegetação típica dos ambientes campestres da Serra da Mantiqueira. Desde então, não houve novas coletas dessa planta.

O presente trabalho tem como objetivos relatar a redescoberta na natureza de $O$. leptophylla, apresentar uma breve caracterização morfológica, realizar a tipificação e discutir seu status de conservação.

Durante expedições do Projeto Orchidstudium (www.orchidstudium.com) para o registro fotográfico das espécies de Orchidaceae de Minas Gerais, exemplares de Octomeria leptophylla foram coletados, fotografados em campo e suas características relevantes foram anotadas. As coleções encontram-se depositadas no herbário da Universidade Federal de Juiz de Fora (CESJ).

O mapa destacando as duas localidades deste registro, além da Pedra Branca, foi confeccionado utilizando-se o Americas Base Map (disponível em http://www.nybg.org/bsci/ digital_maps/) empregando o "software" ArcView GIS $3.2^{\circledR}$.

Octomeria leptophylla Barb. Rodr., Gen. Spec. Orchid. 2: 112. 1881. Tipo: BRASIL. MINAS GERAIS: Les environs de la serra de Caldas, près de Pedra Branca, J. B. Rodrigues s.n. (RB - destruído; Lectotypus, aqui designado, figura A, tab. 203, v. 3 da Iconographie des Orchidées du Brésil, depositado em RB e reproduzida em (Sprunger 1996)).

Fig. 2 a-e

Octomeria teretifolia Barb. Rodr., Gen. Spec. Orchid. 1: 32. 1877. nom. Illeg.

Octandrorchis leptophylla (Barb. Rodr.) Brieger, Trab. Congr. Nac. Bot. (Rio de Janeiro) 26: 43. 1977.

Octomeria leptophylla é uma erva epífita, de até $10 \mathrm{~cm}$ alt., com rizoma inconspícuo, ramicaule cilíndrico, 2,5-3,5 cm compr., delicado, coberto por bainhas tubulares,

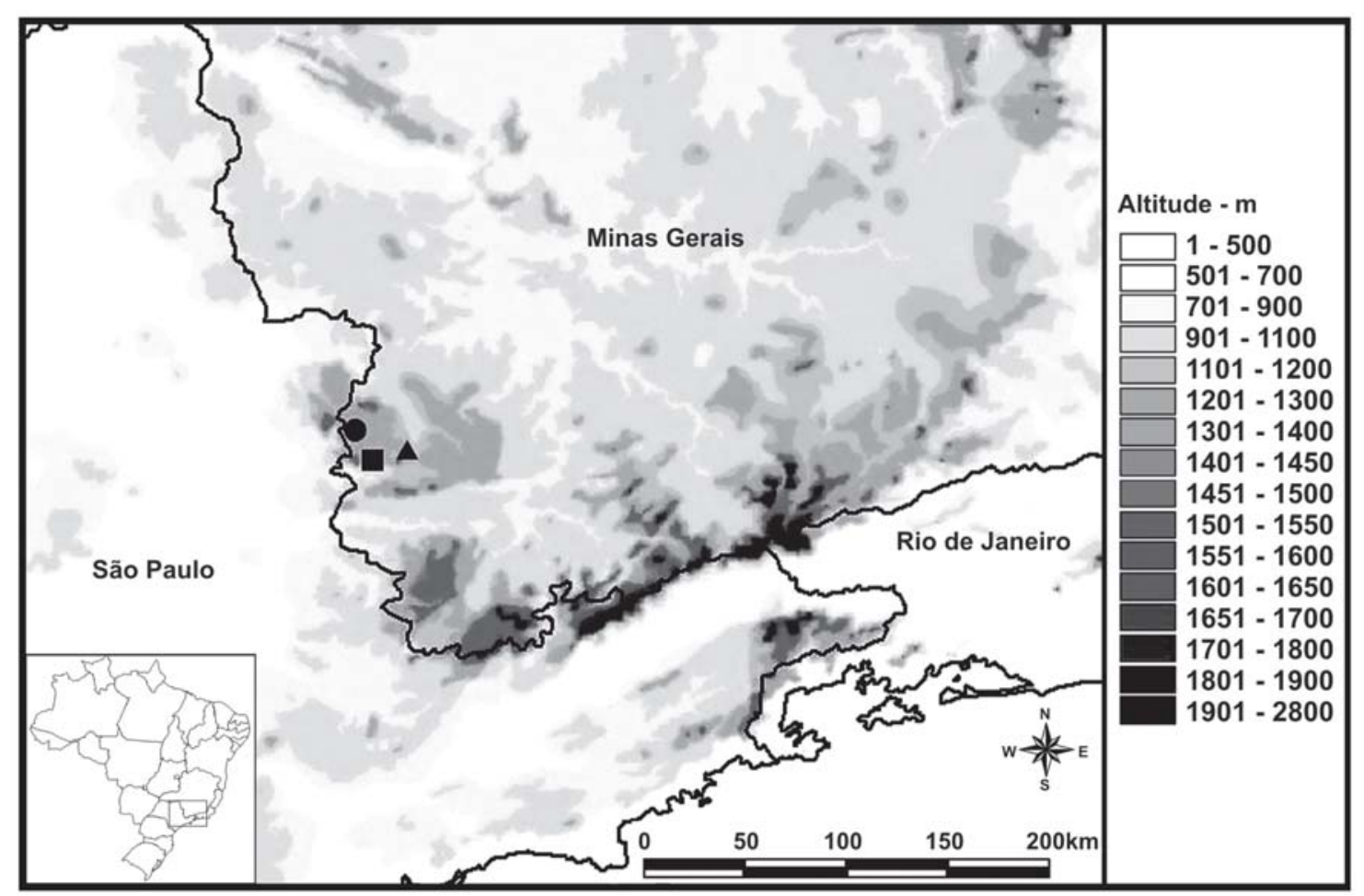

Figura 1 - Localidades de ocorrência de O. leptophylla, Minas Gerais: $(\bullet)$ Morro do Serrote (Poços de Caldas), ( Morro do Mel (Andradas) e ( $\mathbf{\Delta})$ Pedra Branca (Caldas).

Figure 1 - Locations of occurrence of O. leptophylla, Minas Gerais: (•) Morro do Serrote (Poços de Caldas), (ロ) Morro do Mel (Andradas) and ( $\boldsymbol{\Delta})$ Pedra Branca (Caldas). 


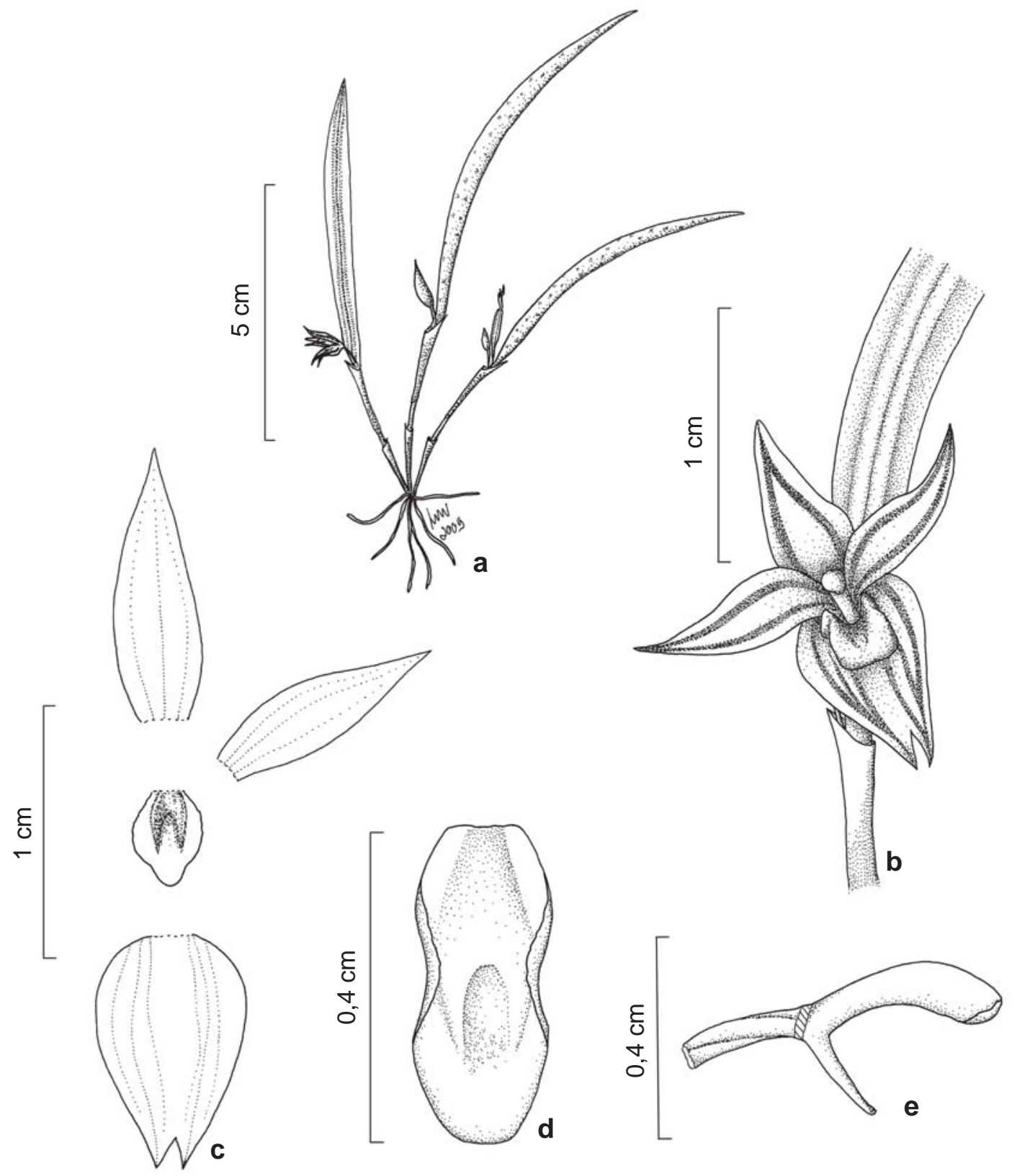

Figura 2 - Octomeria leptophylla - a. hábito; b. flor; c. perianto; d. labelo, vista frontal; e. coluna e ovário, vista lateral.

Figure 2 - Octomeria leptophylla - a. habit; b. flower; c. perianth; d. lip, frontal view; e. column and ovary, lateral view.

estramíneas, portando apenas uma folha semicilíndrica, $4-6 \times$ ca. $0,5 \mathrm{~cm}$, coriácea, levemente curva, verde, pintalgada de vináceo, ápice agudo, a flor surge no ápice do ramicaule, próximo à base da folha, uma por vez, apresentando sépalas e pétalas amarelas, com estrias vináceas, labelo amarelo, com uma mácula atrovinácea cobrindo todo o disco, sépala dorsal lanceolada, ca. 1,1 ×0,35 cm, levemente côncava, ápice agudo, sépalas laterais coalescentes até dois terços do comprimento, ca. $0,9 \times 0,65 \mathrm{~cm}$, de âmbito ovado, ápice agudo, pétalas elípticas, ca. 0,95 $\times 0,3 \mathrm{~cm}$, ápice agudo, labelo trilobado, ca. 0,4 $\times 0,35 \mathrm{~cm}$, levemente recurvado, lobos laterais reniformes, lobo mediano levemente triangular, 
ápice arredondado, coluna creme, ca. 0,4 cm compr., curva, levemente claviforme, portando oito polínias amarelo-claras, claviformes, o fruto é uma cápsula fusiforme, ca. $0,7 \mathrm{~cm}$ compr., costado, com o perianto persistente.

Material examinado: BRASIL. MINAS GERAIS: Município de Andradas, próximo do Morro do Mel, ca. 1450 m alt., $22^{\circ} 01^{\prime}$ S, $43^{\circ} 31^{\prime} \mathrm{W}, 12 . X .2008$, fl., $A$. Docha Neto s. n. (CESJ 52171); Município de Poços de Caldas, próximo da divisa de estado e do Morro do Serrote, ca. $1350 \mathrm{~m}$ alt., $21^{\circ} 53^{\prime} \mathrm{S}, 46^{\circ} 39^{\prime} \mathrm{W}$, 18.X.2008, fl., L. A. Petreca s. n. (CESJ 52172).

Octomeria leptophylla foi uma das muitas novas espécies coletadas e descritas por João Barbosa Rodrigues enquanto esteve no município de Caldas. Foi descrita em 1881, e não houve coletas posteriores durante quase 130 anos. No protólogo, Barbosa Rodrigues faz um comentário vago sobre a localidade de coleta da espécie: "Les environs de la serra de Caldas, près de Pedra Branca". Na Lista de Espécies Ameaçadas de Extinção do Estado de Minas Gerais (Biodiversitas 2007), nove espécies de Orchidaceae são consideradas extintas. Dentre elas figura $O$. leptophylla, conhecida apenas pelo material-tipo. Esta espécie foi reencontrada em dois municípios da região sul do estado de Minas Gerais, Andradas (próximo da localidade denominada Morro do Mel) e Poços de Caldas (próximo da localidade denominada Morro do Serrote) (Fig. 1).

O Morro do Mel é uma área situada nas proximidades da Serra do Caracol (localizada no extremo ocidente da Serra da Mantiqueira, é conhecida pelos moradores da região como Serra de Caldas ou Serra da Pedra Branca), entre as cabeceiras da margem direita do Córrego da Cachoeirinha e a margem esquerda do Córrego da Anta. O Morro do Serrote é uma área circundada por vários cursos d'água, afluentes do Rio das Antas, que forma a Represa do Bortolan. Ambas as localidades são muito semelhantes em termos de topografia e flora, e distam entre si cerca de $16 \mathrm{~km}$ apresentando vegetação de encosta secundária, semidensa, na área populacional da espécie tratada. A despeito de poder ser encontrada como epífita em várias espécies de árvores de médio e grande porte, $O$. leptophylla tem concentração mais acentuada em árvores de Clethra scabra Pers. (Clethraceae), tanto no tronco como também nos ramos superiores, mais iluminados.

Desse modo, os dois exemplares registrados neste trabalho provavelmente são os únicos existentes em herbário atualmente, uma vez que o próprio typus de O. leptophylla, assim como os das demais espécies descritas por João Barbosa Rodrigues são representados pelas ilustrações preparadas por ele, em virtude da destruição deste material após sua morte, possivelmente por enchentes ocorridas no Rio de Janeiro na década de 1940, como relatado por Sprunger (1996).

Aparentemente a espécie está extinta localmente na Pedra Branca, em Caldas, pois não foi mais encontrada nesta região após mais de um século. Essa afirmação é reforçada em virtude de um inventário florístico que está sendo realizado na região por alunos e professores da Universidade Federal de Juiz de Fora, ao longo do último ano e, até o momento, não ter sido observada nesta área. Além disso, nota-se intensa degradação na região da Pedra Branca, em decorrência do turismo desordenado, inclusive com a realização de trilhas por motoqueiros, a despeito da mesma estar incluída em uma Área de Proteção Ambiental, que por definição é uma categoria de unidade de conservação com ocupação humana permitida, não possuindo grande poder de preservação.

Embora a localidade em que ocorrem os exemplares amostrados neste trabalho seja de acesso mais difícil, próximo a uma das localidades (Morro do Mel) existe uma área utilizada por turistas para realização de saltos de vôo livre, denominada Pico do Gavião.

Apesar deste registro demonstrar que a espécie não está extinta, ela ainda pode ser categorizada no status de Criticamente em Perigo (CR), baseado em pelo menos três categorias de ameaça, segundo os critérios 
propostos pela IUCN (2001): degradação e perda do habitat e endemismo extremo.

Esta redescoberta pode ser considerada um episódio feliz na história da biodiversidade brasileira, em que "trazemos novamente à vida" uma espécie que se acreditava estar extinta, mas infelizmente é um episódio isolado, pois a situação real das florestas e campos do país não é tão positiva, uma vez que a fronteira agrícola avança de forma agressiva sobre o cerrado, as madeireiras atuam maciçamente sobre a Amazônia, as mineradoras escavam os campos rupestres e a necessidade de espaço para acomodação da população crescente dizima o pouco que resta da floresta atlântica, de forma mais rápida do que conseguimos estudar estas áreas já tão castigadas pelos cinco séculos de atuação do homem.

Pelo exposto acima, fica clara a relevância dessa redescoberta, e a necessidade de proteção dos vários fragmentos vegetacionais remanescentes dos três biomas que ocorrem em Minas Gerais, sob pena de redução da grande biodiversidade existente no estado e no país como um todo.

Também fica demonstrada a necessidade de mais estudos para um real conhecimento da flora de Minas Gerais, um estado de grandes proporções e com grande variedade de ambientes.

\section{Agradecimentos}

Agradecemos à inestimável contribuição de Lazaro Ademar Petreca, responsável pela redescoberta da espécie e identificação da principal árvore hospedeira da espécie tratada. Também agradecemos a Patricia Harding pelas sugestões e aos dois assessores anônimos que fizeram a revisão deste trabalho.

\section{REFERÊNCIAS BIBLIOGRÁFICAS}

Biodiversitas, 2007. Revisão das listas das espécies da flora e da fauna ameaçadas de extinção do estado de Minas Gerais Relatório final. v.2. Fundação Biodiversitas, Belo Horizonte. Pp 37-104. Disponível em http://www.biodiversitas.org.br/listas-mg

IUCN (International Union for Conservation of Nature and Natural Resources). 2001. IUCN Red List Categories and Criteria, Version 3.1. IUCN Species Survival Commission, Gland and Cambridge, 30p.

Luer, C. A. 1986. Icones Pleurothallidinarum I. Systematics of the Pleurothallidinae (Orchidaceae). Monographs in Systematic Botany from the Missouri Botanical Garden 15: 1-81.

Myers, N.; Mittermeier, R. A.; Mittermeier, C. G.; Fonseca, G. A. B. \& Kent, J. 2000. Biodiversity hotspots for conservation priorities. Nature 403: 853-858.

Pridgeon, A. M. \& Chase, M. W. 2001. A phylogenetic reclassification of Pleurothallidinae (Orchidaceae). Lindleyana 16(4): 235-271.

Pridgeon, A. M; Cribb, P. J.; Chase, M. W. \& Rasmussen, F. N. 2005. Genera Orchidacearum: v.4. Epidendroideae (Part 1). Oxford University Press, Oxford, 696p.

Rodrigues, J. B. 1877. Genera et Species Orchidearum Novarum. v.1. Typographia Nacional, Rio de Janeiro, 206p.

Sprunger, S. (ed.). 1996. João Barbosa Rodrigues - Iconographie des orchidées du Brésil. v.1: The illustrations. Friedrich Reinhardt Verlag, Basle, 540p. 